\title{
SPECTRAL INEQUALITIES INVOLVING THE SUMS AND PRODUCTS OF FUNCTIONS
}

\author{
KONG-MING CHONG \\ Department of Mathematics, University of Malaya \\ Kuala Lumpur 22-11 MALAYSIA
}

(Received April 2, 1975 and in revised form December 11, 1980)

ABSTRACT. In this paper, the notation $\prec$ and $\prec$ denote the Hardy-Littlewood-Polya spectral order relations for measurable functions defined on a finite measure space $(x, \Lambda, \mu)$ with $\mu(x)=a$, and expressions of the form $f<g$ and $f<<g$ are called spectral inequalities. If $f, g \in L^{1}(X, \Lambda, \mu)$, it is proven that, for some $b \geqslant 0$, $\log \left[b+\left(\delta_{f}{ }_{g}\right)^{+}\right] \ll \log \left[b+(f g)^{+}\right] \prec \log \left[b+\left(\delta_{f} \delta_{g}\right)^{+}\right]$whenever $\log ^{+}\left[b+\left(\delta_{f} \delta_{g}\right)^{+}\right] \varepsilon$ $L^{1}([0, a])$, here $\delta$ and $\mathrm{l}$ respectively denote decreasing and increasing rearrangement. With the particular case $b=0$ of this result, the Hardy-Littlewood-PolyaLuxemburg spectral inequality $f g \ll \delta_{f} \delta_{g}$ for $0 \leqslant f, g \in L^{1}(x, \Lambda, \mu)$ is shown to be a consequence of the well-known but seemingly unrelated spectral inequality $f+g \prec \delta_{f}$ $+\delta_{g}$ (where $\left.f, g \in L^{1}(X, \Lambda, \mu)\right)$, thus giving new proof for the former spectral inequa1ity. Moreover, the Hardy-Littlewood-Polya-Luxemburg spectral inequality is also extended to give $\left(\delta_{f}{ }_{g}\right)^{+} \prec(f g)^{+} \longleftrightarrow\left(\delta_{f} \delta_{g}\right)^{+}$and $\left.\left(\delta_{f} \delta_{g}\right)^{-} \prec(f g)^{-} \ll\left(\delta_{f}\right)_{g}\right)^{-}$for not necessarily non-negative $f, g \in L^{1}(X, \Lambda, \mu)$.

KEY WORDS AND PHRASES. Equimeasurable Rearrangements, Spectral Inequalities, Convex functions, discrete measure, non-atomic measure, martingale convergence theorem.

2980 MATHEMATICS SUBJECT CLASSIFICATION CODE. $26 D 20$.

1. INTRODUCTION

In this paper, we prove some spectral inequalities involving the sums and products of measurable functions as well as their equimeasurable rearrangements, With 
these results, we extend some known rearrangement inequalities of Day [1, pp.941-943], Hardy-Littlewood-Pólya [2, Theorem 378, p. 278], London [3, Theorems 1 and 2], Lorentz-Shimogaki [4, Proposition 1, p. 34] and Luxemburg [5, Theorem 8.2, p. 102]. Moreover, using our method of approach, we obtain in several cases more general conditions for equality than those given by either Day in [1] or London in [3].

\section{PRELIMINARIES.}

Let $(X, \Lambda, \mu)$ be a finite measure space with total measure $\mu(X)=a$. Whenever $X$ is clear from the context, we often write $\int . d \mu$ for integration over $X$ for any function with either the positive or negative part integrable. By $M(X, \mu)$ we denote the set of all extended real valued measurable functions on $x$. Two functions $f \varepsilon$ $M(X, \mu)$ and $g \in M\left(X^{\prime}, \mu^{\prime}\right)$, where $\mu^{\prime}\left(X^{\prime}\right)=\mu(X)=a$, are said to be equimeasurable (written $f \sim g$ ) whenever

$$
\mu(\{x: f(x)>t\})=\mu^{\prime}(\{x: g(x)>t\})
$$

for all real $t$. If $f \sim g$, it is not hard to see that

$$
\Phi(\mathrm{f}) \sim \Phi(\mathrm{g})
$$

whenever $\Phi: R \rightarrow R$ is a Borel measurable function.

If $f \varepsilon M(X, \mu)$, it is well-known that there exists a unique right continuous nonincreasing function $\delta_{f}$ on the interval $[0, a]$, called the decreasing rearrangement of $f$, such that $\delta_{f}$ and $f$ are equimeasurable. In fact,

$$
\delta_{f}(s)=\inf \{t \in R: \mu(\{x: f(x)>t\}) \leqslant s\}
$$

for all $s \in[0, a]$. Observe that there also exists a unique right continuous nondecreasing function $l_{f}=-\delta_{-f}$, called the increasing rearrangement of $f$, such that $\mathbf{l}_{\mathbf{f}} \sim \mathbf{f}$.

In what follows, we denote the Lebesgue measure on the real line $R$ by $m$.

It is easy to see that $\delta_{f_{n} \uparrow=1} \uparrow_{f}^{\infty}$ whenever $f_{n} \uparrow f$, where $f_{n=1}^{\infty}$, $f \in M(X, \mu), n=$ $1,2,3, \ldots$. The following theorem generalizes this fact.

THEOREM 2.1 If $\mathrm{f}_{\mathrm{n}} \in \mathrm{M}(\mathrm{X}, \mu)$, where $\mathrm{n}=1,2,3, \ldots$, then

$$
\delta_{1 \operatorname{im} \inf f_{n}}(t) \leqslant \lim \inf \delta_{f_{n}}(t)
$$

and 


$$
\delta_{1 i m \sup } f_{n}\left(t^{-}\right) \geqslant \lim \sup \delta_{f_{n}}\left(t^{-}\right)
$$

for all $t \in[0, a]$. Thus, if $f_{n}$ converges to $f \mu-a . e$ as $n \rightarrow \infty$, then $\delta_{f_{n}}$ converges to $\delta_{f}$ as $n \rightarrow \infty$ at every point of continuity of $\delta_{f}$.

Furthermore, if $f_{n}$ converges to $f$ in measure, then $\delta_{f_{n}}$ also converges to $\delta_{f}$ in measure and $f_{n}$ converges to $f$ in $L^{P}$ if and only if $\delta_{f_{n}}$ converges to $\delta_{f}$ in $\mathrm{L}^{\mathrm{P}}$, where $1 \leqslant \mathrm{p}<\infty$.

PROOF. Equation (2.4) follows from the fact that $\delta_{f_{n}} \stackrel{\infty}{n=1}_{f}^{\infty}$ whenever $f_{n} \underset{n=1}{\uparrow} f$ in exactly the same way that Fatou's Lemma is obtained from Lebesgue's Monotone Convergence Theorem. Next, on applying (2.4) to the functions $\left\{-f_{n}\right\}_{n=1}^{\infty}$ and using the fact that $\delta_{-f}(t)=-\delta_{f}\left((a-t)^{-}\right)$for $t \varepsilon[0, a]$, we immediately obtain $\overline{1 i m} \delta_{f_{n}}$ $\left((a-t)^{-}\right) \leqslant \delta \overline{\lim }_{n}\left((a-t)^{-}\right), t \varepsilon[0, a]$, whence $(2.5)$ follows.

Now, if $f_{n}$ converges to $f$ in measure as $n \rightarrow \infty$, let $\left\{\delta_{f_{n_{i}}}\right\}_{i=1}^{\infty}$ be any subsequence of $\left\{\delta_{f_{n}}\right\}$; then $\left\{f_{n_{i}}\right\}_{i=1}^{\infty}$ is a subsequence of $\left\{f_{n}\right\}$ and hence contains a subsequence $\left\{f_{n_{i_{j}}}\right\}_{j=1}^{\infty}$ which converges to $f \mu-a . e .$, by Riesz's Theorem. Then $\left\{\delta_{f_{n_{i}}}\right\}_{j=1}^{\infty}$ is a subsequence of $\left\{\delta_{f_{n_{i}}}\right\}_{j=1}^{\infty}$ and $\left\{\delta_{f_{n_{i}}}\right\}_{j=1}^{\infty}$ also converges to $\delta_{f}$ m-a. e. as $j \rightarrow \infty$; so by Riesz's Theorem again, we conclude that $\delta_{f_{n}}$ converges to $\delta_{f}$ in measure (cf. $[6,(11.45), \mathrm{p} .163])$.

The last assertion follows directly from the preceding paragraph and the fact that the operation of decreasing rearrangements preserves uniform integrability (see [7, Section 2]).

REMARK. The preservation of $\mathrm{L}^{\infty}$ convergence is also shown to be true in 
COROLLARY 3.5 below using a different method of proof.

If $f, g \in M(X, \mu), M\left(X^{\prime}, \mu^{\prime}\right)$ and $f^{+}, g^{+} \varepsilon L^{1}(X, \mu), L^{1}\left(X^{\prime}, \mu^{\prime}\right)$ where $\mu(X)=$ $\mu^{\prime}\left(X^{\prime}\right)=a<\infty$, then we write $f<<g$ whenever

$$
\int_{0}^{t} \delta_{f} d m \leqslant \int_{0}^{t} \delta_{g} d m \quad t \varepsilon[0, a]
$$

and $f<g$ whenever $f<g$ and $\int_{0}^{a} \delta_{f} d m=\int_{0}^{a} \delta_{g} d m$.

By an argument given in $[8, \mathrm{p} .152]$, we infer that $(2.6)$ is equivalent to requiring

$$
\int(f-t)^{+} \leqslant \int(g-t)^{+}
$$

for all $t \in R$. Moreover, it can be shown (see [9, Proposition 10.2 (iii), p.62], for example) that

$$
\|f\|_{\infty} \leqslant\|g\|_{\infty}
$$

whenever $f<g$ where $g \in L^{\infty}(X, \mu) \cup L^{\infty}\left(X^{\prime}, \mu^{\prime}\right)$.

In the sequel, expressions of the form $f \prec g$ (respectively $f \ll g$ ) are called strong (respectively weak) spectral inequalities;

\section{SPECTRAL INEQUALITIES INVOLVING THE SUMS OF FUNCTIONS.}

In this section, we give simpler proofs for the spectral inequalities obtained by Lorentz-Shimogaki [4, Proposition 1, p. 34] and Day [1, p. 941] and we also extend them to include not necessarily integrable functions defined on a finite measure space.

In what follows, if $x_{x}=\left(x_{1}, x_{2}, \ldots, x_{n}\right) \varepsilon R^{n}$ is any $n$-tuple, then we write $x^{*}=\left(x_{1}^{*}, x_{2}^{*}, \ldots, x_{n}^{*}\right)$ and $x_{\sim}^{\prime}=\left(x_{1}^{\prime}, x_{2}^{\prime}, \ldots, x_{n}^{\prime}\right)$ to denote respectively the decreasing and increasing rearrangement of $\underset{\sim}{x}$ where we have regarded $\underset{\sim}{x}$ as a measurable function defined on a discrete measure space with $n$ atoms of equal measures.

LEMMA 3.1. If $\underset{\sim}{a}=\left(a_{1}, a_{2}, \ldots, a_{n}\right) \varepsilon R^{n}$ and $b=\left(b_{1}, b_{2}, \ldots, b_{n}\right) \varepsilon R^{n}$, then

$$
\sum_{i=1}^{n}\left(a_{i}^{*}+b_{i}^{\prime}\right)^{+} \leqslant \sum_{i=1}^{n}\left(a_{i}+b_{i}\right)^{+} \leqslant \sum_{i=1}^{n}\left(a_{i}^{*}+b_{i}^{*}\right)^{+} \text {. }
$$

PROOF. Without loss of generality, we may assume that $a_{i}=a_{i}^{*}, i=1,2, \ldots, n$. If $1 \leqslant i<j \leqslant n$ and $b_{i}<b_{j}$, then $i t$ is not hard to see that $\left(a_{i}+b_{i}\right)^{+}+\left(a_{j}+\right.$ 
$\left.b_{j}\right)^{+} \leqslant\left(a_{i}+b_{j}\right)^{+}+\left(a_{j}+b_{i}\right)^{+}$. Thus, for each pair of integers $i, j$ such that $1 \leqslant i$ $<j \leqslant n$, whenever $b_{i}<b_{j}$, the middle sum of (3.1) is never decreased on interchanging $b_{i}$ and $b_{j}$. We therefore conclude that the left hand sum is the minimum possible value while the right hand sum is the maximum possible value attainable by the middle sum as $b$ runs through all its rearrangements.

LEMMA 3.2. If $f, g \in L^{1}(x, \mu)$ then

$$
\int_{0}^{a}\left(\delta_{f}+l_{g}\right)^{+} d m \leqslant \int_{X}(f+g)^{+} d \mu \leqslant \int_{0}^{a}\left(\delta_{f}+\delta_{g}\right)^{+} d m
$$

PROOF. We first note that it suffices to prove (3.2) for non-atomic measure spaces by imbedding $(X, \Lambda, \mu)$ in a non-atomic measure space; we refer to [9, pp. 52-54] or [5] for this method. Thus, we assume that $(x, \Lambda, \mu)$ is non-atomic.

To prove (3.2), we need only prove the right hand inequality, the rest is similar. To this end, we first show that there exist sequences of simple functions $\left\{f_{n}: n \geq 1\right\}$ and $\left\{g_{n}: n>1\right\}$ such that both $f_{n} \rightarrow f$ and $g_{n} \rightarrow g$ not only pointwise almost everywhere but also in $L^{1}$. Now since $(X, \Lambda, \mu)$ is non-atomic, there exists a measure preserving map $\sigma: X \rightarrow[0, a]$ such that $f=\delta_{f} \circ \sigma \mu-a . e .[9$, Theorem 6.2, p.49]. For each $n=1,2,3, \ldots$, and for each $i=1,2,3, \ldots, 2^{n}$, let

$$
E_{i}^{(n)}=\sigma^{-1}\left(\left[\frac{(i-1) a}{2^{n}}, \frac{i a}{2^{n}}\right]\right) .
$$

Define a sequence of functions $\left\{f_{n}: n \geq 1\right\}$ by

$$
f_{n}=\sum_{i=1}^{2^{n}}\left(\frac{1}{\mu\left(E_{i}(n)\right.} \int_{E_{i}}(n) f d \mu\right) x_{E_{i}}(n)
$$

For each $n=1,2,3, \ldots$, let $\Lambda_{n}$ denote the $\sigma$ - algebra generated by $\left\{E_{i}(n)\right.$, $\left.i=1,2,3, \ldots, 2^{n}\right\}$ and let $\Lambda_{\infty}$ denote the $\sigma$-algebra generated by $\left.\right|_{n=1} ^{\infty} \Lambda_{n}$ If $\nu(A)=\int_{A} f d \mu, A \varepsilon \Lambda$, then, by the martingale convergence theorem [6, Theorem 20 . 56, p. 369],

$$
f_{n}=\frac{d\left(\nu \mid \Lambda_{n}\right)}{d\left(\mu \mid \Lambda_{n}\right)} \rightarrow \frac{d\left(\nu \mid \Lambda_{\infty}\right)}{d\left(\mu \mid \Lambda_{\infty}\right)}=f \quad \mu-a \cdot e .
$$


as $\mathrm{n} \rightarrow \infty$, where the convergence takes place point-wise almost everywhere with the limit function being $f \mu$-a.e. since $f=\delta_{f} \circ \sigma$ is $\Lambda_{\infty}-$ measurable and $\Lambda_{\infty}=\sigma^{-1}$ (B) where $B$ denotes the Borel $\sigma$-algebra of $[0, a]$. But, by $[9$, Proposition 10 . 9, p. 70], $\mathrm{f}_{\mathrm{n}} \prec \mathrm{f}$ for $\mathrm{n}=1,2,3, \ldots$, and so, by [7, Theorem 5.1, p. 403], $\mathrm{f}_{\mathrm{n}} \rightarrow$ $\mathrm{f}$ in $\mathrm{L}^{1}$ as $\mathrm{n} \rightarrow \infty$. Similarly, there exists a sequence of simple functions $\lg _{\mathrm{n}}$ : $n \geq 1\}$ such that $g_{n} \rightarrow g$ pointwise almost everywhere and also in $L^{1}$ as $n \rightarrow \infty$.

Now, by [1, Lemma 5.5, p. 938], there exist two sequences of simple functions $\left\{f_{n}^{\prime}: n \geq 1\right\}$ and $\left\{g_{n}^{\prime}: n \geqslant 1\right\}$ having the same sets of constancy and with these sets having equal measure such that $\left|f_{n}^{\prime}\right| \leqslant\left|f_{n}\right|,\left|g_{n}^{\prime}\right| \leqslant\left|g_{n}\right|$ and both $f_{n}^{\prime}-f_{n} \rightarrow 0$ and $g_{n}^{\prime}-g_{n} \rightarrow 0$ pointwise almost everywhere as $n \rightarrow \infty$. But then $f_{n}^{\prime} \rightarrow f$ and $g_{n}^{\prime} \rightarrow g$ pointwise almost everywhere as $\mathrm{n} \rightarrow \infty$, and the convergence also takes place in $\mathrm{L}^{1}$ by [7, Theorem 5.1. p. 403] since $\left|f_{n}^{\prime}\right| \leqslant\left|f_{n}\right| \ll|f|$ and $\left|g_{n}^{\prime}\right| \leqslant\left|g_{n}^{\prime}\right| \ll|g|$ by virtue of the fact that $f_{n} \prec f \Rightarrow\left|f f_{n}\right| \ll|f|$ and $g_{n}<g \Rightarrow\left|g_{n}\right| \ll|g| \quad$ (see [9, Theorem 10.10$, p. 71$])$.

Hence, by Lemma 3.1 , we have

$$
\int_{X}\left(f_{n}^{\prime}+g_{n}^{\prime}\right)^{+} d \mu \leqslant \int_{0}^{a}\left(\delta_{f_{n}^{\prime}}+\delta_{g_{n}^{\prime}}\right)^{+} d m
$$

where the result follows by taking limits.

THEOREM 3.3. If $\mathrm{f}, \mathrm{g} \in \mathrm{L}^{1}(\mathrm{X}, \mu)$, then

$$
\delta_{f}+l_{g} \curlywedge f+g<\delta_{f}+\delta_{g}
$$

PROOF. On substituting $f-t$ for $f$ in (3.2), we see that the result follows immediately from Lemma 3.2 , by virtue of (2.7).

REMARK. The spectral inequality $f+g<\delta_{f}+\delta_{g}$ is well-known (see [9, p. 88] and [5, Theorem 10.1, p. 108]) and it can be used [7, Theorem 2.2, p. 397] for giving a simple proof of a result concerning the uniform integrability of functions. On the other hand, the spectral inequality $\delta_{f}+l_{g} \prec f+g$ is obtained by Day in [ 1 , p. 941]. In either case, our present method of approach is simpler. 
COROLLARY 3.4. If $\mathrm{f}, \mathrm{g} \in \mathrm{L}^{1}(\mathrm{X}, \mu)$, then

$$
\delta_{f}-\delta_{g}<f-g<\delta_{f}-l_{g}
$$

PROOF. Since $l_{g}=-\delta_{-g}$, the result follows immediately from Theorem 3.3 on substituting $-\mathrm{g}$ for $\mathrm{g}$ in (3.3).

COROLLARY 3.5. (Lorentz-Shimogaki [4, Proposition 1, p. 34]). If $\mathrm{f}, \mathrm{g} \varepsilon \mathrm{L}^{1}([0$, 1], m) are positive, then

$$
\left|\delta_{f}-\delta_{g}\right| \prec<|f-g|
$$

PROOF. This is an immediate consequence of the preceding corollary and a theorem of Luxemburg [5, Theorem 9.5, p. 107].

REMARK. The notation $\mathrm{f} \prec \mathrm{g}$ used by Lorentz and Shimogaki in [4] means $|\mathrm{f}| \prec \prec$ $|g|$ in our present context.

COROLLARY 3.6. Suppose $\mathrm{f}_{\mathrm{n}} \varepsilon \mathrm{L}^{\infty}(\mathrm{X}, \mu), \mathrm{n}=1,2, \ldots$. If $\mathrm{f}_{\mathrm{n}}$ converges in $L^{\infty}$ to a function $f \in L^{\infty}(X, \mu)$ as $n \rightarrow \infty$, then $\delta_{f_{n}}$ also converges in $L^{\infty}$ to $\delta_{f}$ as $n \rightarrow$ $\infty$.

PROOF. Using Corollary 3.4, we have, for each $n=1,2,3, \ldots, \delta_{f}-\delta_{f}<f_{n}-$ f and so, by $(2.8),\left\|\delta_{f}-\delta_{f}\right\|_{\infty} \leqslant\|f(f)\|_{\infty}$ whence the result follows.

The following simple theorem is useful especially for generalizing certain rearrangement inequalities (by removing restrictions that certain functions must be nonnegative) and certain spectral inequalities (by removing restrictions that certain functions must be integrable).

THEOREM 3.7. If $\mathrm{f} \varepsilon \mathrm{L}^{1}(\mathrm{x}, \mu)$, then the sequence $\left\{\mathrm{f}_{\mathrm{n}}\right\}_{\mathrm{n}=0}^{\infty}$ defined by $\mathrm{f}_{\mathrm{n}}=(\mathrm{f}+$ $n)^{+}-n$ satisfies $\left|f_{n}\right| \leqslant|f|$ for all $n$ and decreases to $f$ as $n \rightarrow \infty$. Consequent1y $\int f_{n} d \mu \underset{n=0}{\downarrow} \int_{n}^{\infty} d \mu$.

In general, if $\mathrm{f} \varepsilon \mathrm{M}(\mathrm{X}, \mu)$ with $\mathrm{f}^{+} \varepsilon \mathrm{L}^{1}(\mathrm{X}, \mu)$, then, as $\mathrm{n} \rightarrow \infty, \mathrm{f}_{\mathrm{n}} \downarrow \mathrm{f}$ and $\int \mathrm{f}_{\mathrm{n}}$

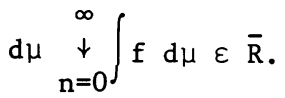

PROOF. The result follows immediately from Lebesgue's Dominated Convergence Theo- 
rem. and Levi's Monotone Convergence Theorem.

The following theorem extends Theorem 3.3 to include measurable functions which are not necessarily integrable.

THEOREM 3.8. Suppose $\mathrm{f}, \mathrm{g} \varepsilon \mathrm{M}(\mathrm{X}, \mu)$. If $\mathrm{f}^{+}, \mathrm{g}^{+} \varepsilon \mathrm{L}^{1}(\mathrm{X}, \mu)$, then

$$
\delta_{f}+l_{g}<f+g<\delta_{f}+\delta_{g}
$$

or, equivalently, if $\mathrm{f}^{+}, \mathrm{g}^{-} \varepsilon \mathrm{L}^{1}(\mathrm{x}, \mu)$, then

$$
\delta_{f}-\delta_{g}<f-g<\delta_{f}-l_{g}
$$

PROOF. If suffices to prove $\delta_{f}+l_{g}<f+g$, the rest is similar. Let $f_{n}=$ $(f+n)^{+}-n, g_{n}=(g+n)^{+}-n$, where $n=0,1,2, \ldots$. Then $\delta_{f}=\left(\delta_{f}+n\right)^{+}-n$, $l_{g_{n}}=\left(l_{g}+n\right)^{+}-n$. Clearly, $f_{n}, g_{n} \varepsilon L^{1}(x, \mu)$ for $n=0,1,2, \ldots$ and so $\delta_{f}+$ $\mathrm{l}_{\mathrm{n}}<\mathrm{f}_{\mathrm{n}}+\mathrm{g}_{\mathrm{n}}$ by Theorem 3.3. But, as $\mathrm{n} \rightarrow \infty, \delta_{f_{n}}+\mathrm{l}_{\mathrm{g}_{\mathrm{n}}} \downarrow \delta_{\mathrm{f}}+\mathrm{l}_{\mathrm{g}}, \mathrm{f}_{\mathrm{n}}+\mathrm{g}_{\mathrm{n}} \downarrow \mathrm{f}+\mathrm{g}$ by Theorem 3.7. Hence $\delta_{f}+l_{g} \prec f+g$, by $[10$, Corollary 1.11].

COROLLARY 3.9. Suppose $\mathrm{f}_{1}, \mathrm{f}_{2}, \ldots, \mathrm{f}_{\mathrm{n}} \varepsilon \mathrm{M}(\mathrm{X}, \mu)$. If $\mathrm{f}_{\mathrm{i}}^{+} \varepsilon \mathrm{L}^{1}(\mathrm{X}, \mu), i=1,2, \ldots$ ., $\mathrm{n}$, then

$$
\mathrm{f}_{1}+\mathrm{f}_{2}+\ldots+\mathrm{f}_{\mathrm{n}}<\delta_{\mathrm{f}_{1}}+\delta_{\mathrm{f}_{2}}+\ldots+\delta_{\mathrm{f}_{\mathrm{n}}}
$$

PROOF. The result follows from Theorem 3.8 by induction.

As a direct consequence of Theorem 3.8 above, the following theorem proves some basic spectral inequalities which can be used to give simple proofs for some known spectral inequalities involving the products of functions.

THEOREM 3.10. If $f, g \in L^{1}(X, \mu)$ are either both non-negative or both non-positive, then

$$
\log \delta_{f} l_{g}<\log f g<\log \delta_{f} \delta_{g}
$$

In general, if $\mathrm{f}_{1}, \mathrm{f}_{2}, \ldots, \mathrm{f}_{\mathrm{n}} \varepsilon \mathrm{L}^{1}(\mathrm{x}, \mu)$ are all non-negative, then

$$
\log \mathrm{f}_{1} \mathrm{f}_{2} \ldots \mathrm{f}_{\mathrm{n}}<\log \delta_{\mathrm{f}_{1}} \delta_{\mathrm{f}_{2}} \ldots \delta_{\mathrm{f}_{\mathrm{n}}}
$$

PROOF. If $f, g \in \mathrm{L}^{1}(\mathrm{X}, \mu)$ are both non-negative, then the result follows immediately from Theorem 3.8 on replacing $f, g$ respectively by $\log f$, $10 g$ and on using 
the fact that $\delta_{\log f}=\log \delta_{f}, \log \delta_{g}=\delta_{\log g}$ and $(\log f g)^{+}=(\log f+\log g)^{+} \leqslant$ $\log ^{+} f+\log ^{+} g \leqslant f+g \varepsilon L^{1}(X, \mu)$.

The case that both $f, g \in L^{1}(X, \mu)$ are non-positive follows immediately by applying the previous case to $-f$ and $-g$, and using [9, Theorem 4.6, p. 33].

\section{SOME SPECTRAL INEQUALITIES INVOLVING THE PRODUCTS OF FUNCTIONS}

The following theorem extends some spectral inequalities obtained by Luxemburg [5, Theorem 10.4] (cf. [9, p. 88]) and Day [1, p. 942] to include not necessarily nonnegative integrable functions. Using this extension, we can easily derive Luxemburg's generalization [5, Theorem 8.2, p. 102] of a theorem of Hardy-Littlewood-Pólya [2, Theorem 378, p. 278] . Furthermore, our method enables us to give a further extension of Hardy-Littlewood-Pólya-Luxemburg's Theorem using increasing functions of mixed convex-concave type (see Theorem 4.2 below).

THEOREM 4.1. Suppose $f, g \in L^{1}(X, \mu)$. If $\left(\delta_{f} \delta_{g}\right)^{+} \varepsilon L^{1}([0, a], m)$ (respectively $\left.\left(\delta_{f^{l}}\right)^{-} \varepsilon L^{l}([0, a], m)\right)$, then

$$
\left(\delta_{f l}\right)^{+}<<(f g)^{+}<<\left(\delta_{f} \delta_{g}\right)^{+}
$$

(respectively $\left.\left(\delta_{f} \delta_{g}\right)^{-} \prec \prec(f g)^{-} \ll\left(\delta_{f l}\right)^{-}\right)$and the strong spectral inequalities $\left(\delta_{f} g\right)^{+}<(f g)^{+} \prec\left(\delta_{f} \delta_{g}\right)^{+} \quad$ (respectively $\left(\delta_{f} \delta_{g}\right)^{-} \prec(f g)^{-}<\left(\delta_{f l} g^{-}\right)$hold if and only if $\left(\delta_{f} g_{g}\right)^{+} \sim(f g)^{+} \sim\left(\delta_{f} \delta_{g}\right)^{+}$(respectively $\left.\left(\delta_{f} \delta_{g}\right)^{-} \sim(f g)^{-} \sim\left(\delta_{f} g\right)^{-}\right)$.

Furthermore, $\Phi\left[\left(\delta_{f l}\right)^{+}\right] \ll \Phi\left[(f g)^{+}\right] \ll \Phi\left[\left(\delta_{f} \delta_{g}\right)^{+}\right] \quad\left(\right.$ respectively $\Phi\left[\left(\delta_{f} \delta_{g}\right)^{-}\right] \ll<$ $\Phi\left[(\mathrm{fg})^{-}\right] \ll \Phi_{\left.\left.\left(\mathrm{fl}_{\mathrm{g}}\right)^{-}\right]\right)}$for all increasing convex functions $\Phi: \mathrm{R}^{+} \rightarrow \mathrm{R}$ such that $\Phi^{+}\left[\left(\delta_{f} \delta_{g}\right)^{+}\right] \varepsilon L^{1}([0, a], m) \quad\left(\right.$ respectively $\left.\Phi^{+}\left[\left(\delta_{f^{l}}\right)^{-}\right] \varepsilon L^{1}([0, a], m)\right)$. If, in addition, $\Phi$ is strictly convex and increasing such that $\Phi\left[\left(\delta_{f} \delta_{g}\right)^{+}\right] \varepsilon L^{1}([0, a]$, m) (respectively $\left.\Phi\left[\left(\delta_{f^{l} g}\right)^{-}\right] \varepsilon L^{1}([0, a], m)\right)$, then the strong spectral inequalities $\Phi\left[\left(\delta_{f^{l} g}\right)^{+}\right.$ ]$<\Phi\left[(\mathrm{fg})^{+}\right]<\Phi\left[\left(\delta_{f} \delta_{g}\right)^{+}\right] \quad$ (respectively $\Phi\left[\left(\delta_{f} \delta_{g}\right)^{-}\right]<\Phi\left[(f g)^{-}\right]<\Phi\left[\left(\delta_{f} g^{-}\right]\right.$) hold if and only if $\left(\delta_{f^{l} g}\right)^{+} \sim(f g)^{+} \sim\left(\delta_{f} \delta_{g}\right)^{+} \quad\left(\right.$ respectively $\left.\left(\delta_{f} \delta_{g}\right)^{-} \sim(f g)^{-} \sim\left(\delta_{f l}\right)^{-}\right)$.

PROOF. We first prove the theorem for the case that $f \geqslant 0$ and $g \geqslant 0$. In this case, (3.9) holds. 
Assume $f g \in L^{1}(X, \mu)$. Since the exponential function $\exp : R \rightarrow R^{+}$is (increasing and strictly) convex and since $\exp (\log f g)=f g \varepsilon L^{1}(X, \mu)$, we have, on applying [10, Theorem 3.3] to the left spectral inequality of (3.9), $\exp \left(\log _{f} \delta_{g}\right)$ ২i exp $(\log f g)$, i.e., $\delta_{f} g_{g} \prec f g$. The spectral inequality $f g \prec \delta_{f} \delta_{g}$ is proved analogously. Again, if $f g \in L^{1}(X, \mu)$, then $\log (1+f g) \varepsilon L^{1}(X, \mu)$ and, since the function $t \rightarrow$ $\log \left(1+e^{t}\right), t \varepsilon R, \quad$ is increasing and convex, by Theorem 3.10 and [10, Theorem 3.3], we have $\log \left(1+\delta_{f} l_{g}\right) \ll \log (1+f g)$. Thus, by [10, Corollary 2.4], the strong spectral inequality $\delta_{f^{l}} \prec f g$ or, equivalently, $1+\delta_{f} l_{g} \prec 1+f g, i . e ., \exp (\log (1+$ $\left.\left.\delta_{f^{l}} g\right)\right)<\exp (\log (1+f g))$, holds if and only if $\exp \left(\log \left(1+\delta_{f^{l}}\right)\right) \sim \exp (\log (1+f g))$, i.e., $1+\delta_{f} l_{g} \sim 1+f g$ or, equivalently, $\delta_{f} l_{g} \sim f g$. Similarly, if $\delta_{f} \delta_{g} \varepsilon L^{1}([0, a]$, $\mathrm{m})$, then $\mathrm{fg}<\delta_{\mathrm{f}} \delta_{\mathrm{g}}$ and $\mathrm{fg} \sim \delta_{\mathrm{f}} \delta_{\mathrm{g}}$ are equivalent.

By [10, Theorem 3.3], it is clear that $\Phi\left(\delta_{f l g}\right) \ll \Phi(f g)$ (respectively $\Phi(f g) \prec<$ $\left.\Phi\left(\delta_{f} \delta_{g}\right)\right)$ for all non-decreasing convex functions $\Phi: R^{+} \rightarrow R$ such that $\Phi^{+}(f g) \varepsilon L^{1}$ $(\mathrm{X}, \mu)$ (respectively $\Phi^{+}\left(\delta_{f} \delta_{g}\right) \varepsilon L^{1}([0, a], m)$ ). The last assertion is an immediate consequence of [10, Theorem 3.3] and the foregoing result.

To prove the theorem in general, we need only establish the spectral inequality $\left(\delta_{f} l_{g}\right)^{+} \ll(f g)^{+}$and the corresponding assertions concerning the strong spectral inequality $\left(\delta_{f} l_{g}\right)^{+}<(f g)^{+}$, the rest is analogous. To this end, we first note that $\left(\delta_{f^{l} g}\right)^{+}=\delta_{f^{+}} l_{g^{+}}+l_{f^{-}} \delta_{g^{-}}\left(\right.$since $\delta_{f}=\delta_{f^{+}}-l_{f^{-}}$and $\left.l_{f}=l_{f^{+}}-\delta_{f^{-}}\right)$and (fg) $=$ $\mathrm{f}^{+} \mathrm{g}^{+}+\mathrm{f}^{-} \mathrm{g}^{-}$where the summands on the right in each expression have disjoint supports.

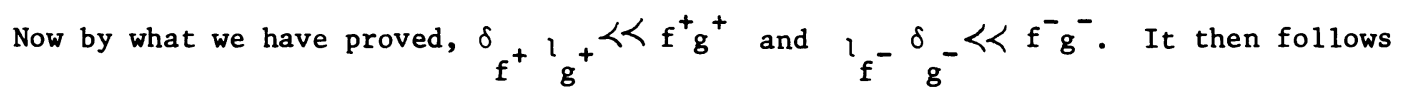

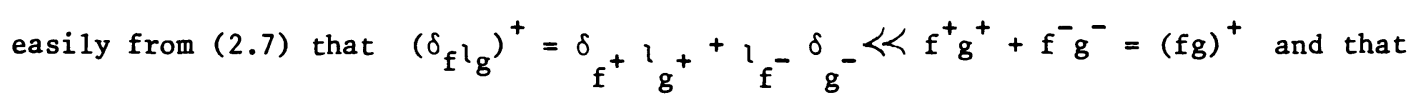
$\left(\delta_{\mathrm{f}^{l} \mathrm{~g}}\right)^{+} \prec(\mathrm{fg})^{+}$if and only if $\delta_{\mathrm{f}^{+}}{ }^{\mathrm{l}} \mathrm{g}^{+}\left\langle\mathrm{f}^{+} \mathrm{g}^{+}\right.$and ${ }_{\mathrm{f}^{-}} \delta_{\mathrm{g}^{-}} \prec \mathrm{f}^{-} \mathrm{g}^{-}$. But, by the result established in the preceding paragraph, $\delta_{\mathrm{f}^{+}} \mathrm{l}_{\mathrm{g}}^{+} \prec \mathrm{f}^{+} \mathrm{g}^{+}$and $\mathrm{l}_{\mathrm{f}^{-}}^{-} \delta_{\mathrm{g}^{-}} \succ^{-} \mathrm{f}^{-} \mathrm{g}^{-}$are respectively equivalent to $\delta_{\mathrm{f}^{+}} \mathrm{l}_{\mathrm{g}}+\sim \mathrm{f}^{+} \mathrm{g}^{+}$and ${ }_{\mathrm{f}} \mathrm{f}_{\mathrm{g}} \delta_{\mathrm{g}^{-}} \sim \mathrm{f}^{-} \mathrm{g}^{-}$. 
Thus, using (2.7) again, we conclude that $\delta \mathrm{f}^{+} \mathrm{l}_{\mathrm{g}}^{+}+\mathrm{f}^{+} \mathrm{g}^{+}$and $\mathrm{l}_{\mathrm{f}^{-}}{ } \mathrm{g}^{-} \mathrm{f}^{-} \mathrm{g}^{-}$if and only if $\delta_{f^{+}} l_{g^{+}}+l_{f^{-}} \delta_{g^{-}} \sim \mathrm{f}^{+} \mathrm{g}^{+}+\mathrm{f}^{-} \mathrm{g}^{-}$or $\left(\delta_{\mathrm{fl}^{l} \mathrm{~g}^{+}} \sim(\mathrm{fg})^{+}\right.$.

REMARK. In theorem 4.1, the spectral inequality $f g \ll \delta_{f} \delta_{g}$ (where $0 \leqslant f, g \varepsilon$ $\left.L^{1}(X, \mu)\right)$ is shown to be a consequence of the wel1-known but seemingly unrelated spectral inequality $f+g<\delta_{f}+\delta_{g}$ (where $f, g \in L^{1}(X, \mu)$ ), thus giving new proof for the former spectral inequality.

THEOREM 4.2. If $\mathrm{f}, \mathrm{g} \in \mathrm{M}(\mathrm{X}, \mu)$, then

$$
\int_{0}^{a} \Phi\left(\delta_{f^{l}}\right) d m \leqslant \int_{X} \Phi(f g) d \mu \leqslant \int_{0}^{a} \Phi\left(\delta_{f} \delta_{g}\right) d m
$$

for all increasing functions $\Phi: R \rightarrow R$ which vanishes at $0 \varepsilon R$ and which is concave on $(-\infty, 0]$ and convex on $[0, \infty)$.

If, in addition, $\Phi$ is strictly increasing such that $\Phi\left(\delta_{f} \delta_{g}\right) \varepsilon L^{1}([0, a], m)$ (respectively $\Phi\left(\delta_{f^{l}}\right) \in L^{1}([0, a], m)$ ), then $\int_{X} \Phi(f g) d \mu=\int_{0}^{a} \Phi\left(\delta_{f} \delta_{g}\right) d m \quad$ (respectively $\left.\int_{0}^{a} \Phi\left(\delta_{f^{l}}\right) d m=\int_{X} \Phi(f g) d \mu\right)$ if and only if $f g \sim \delta_{f} \delta_{g} \quad\left(\right.$ respectively $\left.\delta_{f^{l}} \sim f g\right)$. PROOF. We may assume that $\Phi$ is non-constant and that both $\Phi^{+}\left(\delta_{f} \delta_{g}\right)$ and $\Phi^{-}\left(\delta_{f} \delta_{g}\right)$ are integrable, otherwise, there is nothing to prove. Since $\Phi^{+}: R \rightarrow R^{+}$, $\Phi\left[\mathrm{R}^{+}\right] \mathrm{R}^{+}$and $\Phi(0)=0$, it is easy to see that $\Phi^{+}\left(\delta_{f} \delta_{g}\right)=\Phi\left[\left(\delta_{f} \delta_{g}\right)^{+}\right]$. Since $\Phi$ is convex, non-negative and increasing on $[0, \infty)$, it is also easy to see that $\left(\delta_{\mathrm{f}} \delta_{\mathrm{g}}\right)^{+} \varepsilon \mathrm{L}^{1}\left([0, \mathrm{a}]\right.$, m) whenever $\Phi\left[\left(\delta_{\left.\mathrm{f}_{\mathrm{g}} \delta\right)}{ }^{+}\right] \varepsilon \mathrm{L}^{1}([0, \mathrm{a}], \mathrm{m})\right.$. Similarly, $\Phi^{-}\left(\delta_{\mathrm{f}^{\mathrm{l}} \mathrm{g}}\right) \varepsilon$ $\mathrm{L}^{1}([0, \mathrm{a}], \mathrm{m})$ implies $\left(\delta_{\mathrm{f}^{\mathrm{l}} \mathrm{g}}\right)^{-} \varepsilon \mathrm{L}^{1}([0, \mathrm{a}], \mathrm{m})$, by noting that $\Phi^{-}\left(\delta_{\mathrm{f}^{l} \mathrm{~g}}\right)=-\Phi\left[-\left(\delta_{\mathrm{f}^{l} \mathrm{~g}}\right)^{-}\right]$ and that the function $t \rightarrow-\Phi(-t)$ is convex, non-negative and increasing in $t \in \mathrm{R}^{+}$. Thus, by Theorem 4.1, we have $\left.\Phi\left[\left(\delta_{\mathrm{fl}^{l}}\right)^{+}\right] \ll \Phi\left[(\mathrm{fg})^{+}\right] \ldots{ }^{+}{ }^{+}\left(\delta_{\mathrm{f}} \delta_{\mathrm{g}}\right)^{+}\right]$ and $-\Phi\left[-\left(\delta_{f} \delta_{g}\right)^{-}\right]<^{\prime}-\Phi\left[-(f g)^{-}\right] \cdots-\Phi\left[-\left(\delta_{f l}\right)^{-}\right]$which imply that $\int_{0}^{a} \Phi\left[\left(\delta_{f} l_{g}\right)^{+}\right] d m \leqslant$ $\int_{X} \Phi\left[(f g)^{+}\right] d \mu \leqslant \int_{0}^{a} \Phi\left[\left(\delta_{f} \delta_{g}\right)^{+}\right] d m$ and $\int_{0}^{a} \Phi\left[-\left(\delta_{f l}\right)^{-}\right] d m \leqslant \int_{X} \Phi\left[-(f g)^{-}\right] d \mu \leqslant \int_{0}^{a} \Phi\left[-\left(\delta_{f} \delta_{g}\right)^{-}\right]$ $\mathrm{dm}$, whence the required inequalities follow easily.

To prove the final assertions concerning the case of equalities, let $\int_{X} \Phi(f g) d \mu$ 
$=\int_{0}^{a} \Phi\left(\delta_{f} \delta_{g}\right) d m$ for some strictly increasing function $\Phi$ satisfying the given hypotheses. Then, since $\left.\int_{X} \Phi(f g) d \mu=\int_{X} \Phi\left[(f g)^{+}\right] d \mu+\int_{X} \Phi H(f g)^{-}\right] d \mu \leqslant \int_{0}^{a} \Phi\left[\left(\delta_{f \delta}\right)^{+}\right] d m+$ $\int_{0}^{a} \Phi\left[-\left(\delta_{f} \delta_{g}\right)^{-}\right] d m=\int_{0}^{a} \Phi\left(\delta_{f} \delta_{g}\right) d m$, and $\int_{X} \Phi\left[(f g)^{+}\right] d \mu \leqslant \int_{0}^{a} \Phi\left[\left(\delta_{f} \delta_{g}\right)^{+}\right] d m$ and $\int_{X} \Phi\left[-(f g)^{-}\right]$ $\mathrm{d} \mu \leqslant \int_{0}^{\mathrm{a}} \Phi\left[-\left(\delta_{\mathrm{f}} \delta_{\mathrm{g}}\right)^{-}\right] \mathrm{dm}$, we have equalities in the 1ast two inequalities; this is the case if and only if $\Phi\left[\left(f_{g}\right)^{+}\right]<\Phi\left[\left(\delta_{f} \delta_{g}\right)^{+}\right]$and $-\Phi\left[-\left(\delta_{f} \delta_{g}\right)^{-}\right]<-\Phi\left[-(f g)^{-}\right]$, i.e., if and only if $(\mathrm{fg})^{+} \sim\left(\delta_{f_{g} \delta}\right)^{+}$and $\left(\delta_{f} \delta_{g}\right)^{-} \sim(f g)^{-}$, by Theorem 4.1 or, equivalently, $f g \sim \delta_{f} \delta_{g}$, a fact which is easily seen using (2.7).

The rest follows analogously.

REMARK. When $\Phi(t)=t$, Theorem 4.2 gives Hardy-Littlewood-Pólya-Luxemburg's Theorem [5, Theorem 8.2, p. 102] as an important particular case. Moreover, our method shows that the condition imposed earlier by Luxemburg on the functions $f$ and $g$, $i$. e., $\left.\delta_{|f|}\right|^{\delta}|g| \varepsilon L^{1}([0, a], m)$, is not necessary for the theorem to hold, though it is a necessary and sufficient condition to ensure the integrability of both $\delta_{f} l_{g}$ and $\delta_{f} x$ $\delta_{g}$. In our version of the theorem, we have, therefore, omitted this condition, provided the inequality is interpreted in its appropriate sense.

\section{A REARRANGEMENT THEOREM INVOLVING THE SUMS OF FUNCTIONS.}

The following extension of Corollary 3.6 also contains the inequality of HardyLittlewood-Pólya [2, Theorem 378, p. 278] and Luxemburg [5, Theorem 8.2, p. 102] as a particular case.

THEOREM 5.1. If $\mathrm{f}, \mathrm{g} \in \mathrm{L}^{1}(\mathrm{X}, \mu)$, then $\Phi\left(\delta_{\mathrm{f}}+\mathrm{l}_{\mathrm{g}}\right) \ll<(\mathrm{f}+\mathrm{g})$ (respectively $\left.\Phi(f+g)<-\Phi\left(\delta_{f}+\delta_{g}\right)\right)$ for all convex functions $\Phi: R \rightarrow R$ such that $\Phi^{+}(f+g) \varepsilon$ $L^{1}(X, \mu)$ (respectively $\left.\Phi^{+}\left(\delta_{f}+\delta_{g}\right) \varepsilon L^{l}([0, a], m)\right)$ and, more generally, $\int_{0}^{a} \Phi\left(\delta_{f}+l_{g}\right) d m$ $\leqslant \int_{X} \Phi(f+g) d \mu \leqslant \int_{0}^{a} \Phi\left(\delta_{f}+\delta_{g}\right) d m$ for all convex functions $\Phi: R \rightarrow R$.

If, in addition, $\Phi$ is strictly convex such that $\Phi\left(\delta_{f}+\delta_{g}\right) \varepsilon L^{1}([0, a], m)$ (respectively $\left.\Phi(f+g) \varepsilon L^{1}(X, \mu)\right)$, then the strong spectral inequality 
$\Phi(f+g)<\Phi\left(\delta_{f}+\delta_{g}\right) \quad\left(\right.$ respectively $\Phi\left(\delta_{f}+l_{g}\right) \prec \Phi(f+g)$ ) holds if and only if $f+g \sim \delta_{f}+\delta_{g} \quad$ (respectively $\left.\delta_{f}+l_{g} \sim f+g\right)$

PROOF. This is an easy consequence of Theorem 3.8 and [10, Theorem 3.3].

COROLLARY 5.2. If $f, g \in L^{2 n}(x, \mu)$, where $n \geqslant 1$ is an integer, then

$$
\int_{0}^{a}\left(\delta_{f}+l_{g}\right)^{2 n} d m \leqslant \int_{X}(f+g)^{2 n} d \mu \leqslant \int_{0}^{a}\left(\delta_{f}+\delta_{g}\right)^{2 n} d m \text {. }
$$

There is equality on the left (respectively on the right) if and only if $\delta_{f}+l_{g}$ $\sim f+g$ (respectively $\left.f+g \sim \delta_{f}+\delta_{g}\right)$.

PROOF. The result follows easily since the function $\Phi(t)=t^{2 n}$ is strictly convex on $R$.

COROLLARY 5.3. (Hardy-Littlewood-Pólya-Luxemburg). If $\mathrm{f}, \mathrm{g} \varepsilon \mathrm{L}^{1}(\mathrm{X}, \mu)$, then

$$
\int_{0}^{a} \delta_{f^{l} g} d m<\int_{X} f g d \mu \leqslant \int_{0}^{a} \delta_{f} \delta_{g} d m
$$

If $f, g \in L^{2}(X, \mu)$, then equality on the left (respectively on the right) occurs if and only if $\delta_{f}+l_{g} \sim f+g$ (respectively $f+g \sim \delta_{f}+\delta_{g}$ ).

PROOF. If $f, g \in L^{2}(X, \mu)$, then Corollary 5.2 implies

$$
\int_{0}^{a}\left(\delta_{f}+l_{g}\right)^{2} d m \leqslant \int_{X}(f+g)^{2} d \mu \leqslant \int_{0}^{a}\left(\delta_{f}+\delta_{g}\right)^{2} d m
$$

which, after expansions, clearly simplify to give

$$
\int_{0}^{a} \delta_{f l g} d m \leqslant \int_{X} f g d \mu \leqslant \int_{0}^{a} \delta_{f} \delta g d m
$$

where the conditions for equalities'are as stated above.

Since $\delta_{f \wedge t}=\delta_{f} \wedge t, l_{g} \wedge t=l_{g} \wedge t, t \varepsilon R^{+}$, the inequalities just proven can be easily extended to non-negative integrable functions $f, g$ by approximations. The result then follows from the approximation procedure given in Theorem 3.7 .

COROLLARY 5.4. If $\mathrm{f}, \mathrm{g} \varepsilon \mathrm{L}^{2}(\mathrm{X}, \mu)$ where $\mu(\mathrm{X})=\mathrm{a}<\infty$, then $\delta_{\mathrm{f}} \mathrm{g} \sim \mathrm{fg}$ (respectively $f g \sim \delta_{f} \delta_{g}$ ) if and only if $\delta_{f}+l_{g} \sim f+g$ (respectively $f+g \sim \delta_{f}+\delta_{g}$ ).

PROOF. This is a direct consequence of Theorem 4.2 and Corollary 5.3. 
REMARK. The spectral inequality $|f+g|^{P}=\left|\delta_{f}+\delta_{g}\right|^{p}, p \geqslant 1$, which is obtained by putting $\Phi(t)=|t|^{P}$ in Theorem 5.1, can be used for establishing the sub-additivity property of the norm for the Lorentz space $\Lambda(\phi, p)[4]$ (see $[9, p .88]$ ).

Furthermore, Theorem 5.1 also contains the results obtained by Day in $[1, \S 6.4$ and $\$ 6.6, \mathrm{pp} .942-943]$ as particular cases.

\section{AN EXTENSION OF A THEOREM OF LONDON.}

In [3, Theorem 1 and 2], London proved two rearrangement inequalities involving n-tuples and, in [1, p. 943], Day obtained some continuous versions of these theorems. It turns out that [3, Theorem 2] is also a particular case of Theorem 4.1. We shall now give a further generalization of [3, Theorem 1$]$. In either theorem, we obtain conditions for equality for a wider class of convex functions than the one given in [1] or [3], for every strictly convex and increasing function is strictly increasing and convex but not necessarily conversely, e.g., the identity function on $\mathrm{R}$ is strictly increasing and convex but not strictly convex.

THEOREM 6.1. Let $f, g \in \mathrm{L}^{1}(\mathrm{X}, \mu)$ be either both non-positive or both non-negative. Let $\Phi: R^{+} \rightarrow R$ be a function such that $\Phi\left(e^{t}\right)$ is convex and non-decreasing in $t \varepsilon R$. If $\Phi^{+}\left\{c+\left[b+\left(\delta_{f} \delta_{g}\right)^{r}\right]^{\frac{1}{r}}\right\} \varepsilon L^{1}([0, a], m)$ for some $r>0, b \geqslant 0, c \geqslant 0$, then $\Phi\left\{c+\left[b+\left(\delta_{f} g\right)^{r}\right]^{\frac{1}{r}}\right\} \cdots \propto\left\{c+\left[b+(f g)^{r}\right]^{\frac{1}{r}}\right\} \prec \Phi\left\{c+\left[b+\left(\delta_{f} \delta_{g}\right)^{r}\right]^{\frac{1}{r}}\right\}$.

If, in addition, $\Phi\left(e^{t}\right)$ is strictly increasing and convex in $t \varepsilon R$ such that $\Phi\left\{c+\left[b+(f g)^{r}\right]^{\frac{1}{r}}\right\} \varepsilon L^{1}(X, \mu) \quad\left(\right.$ respectively $\Phi\left\{c+\left[b+\left(\delta_{f} \delta_{g}\right)^{r}\right]^{\frac{1}{r}}\right\} \varepsilon L^{1}([0, a], m)$ ) and $b+c>0$, then the left (respectively right) spectral inequality is strong if and only if $\delta_{f l g}$ and $f g$ (respectively $f g$ and $\delta_{f} \delta_{g}$ ) are equimeasurable.

PROOF. This follows directly from [10, Theorem 3.3, p. 1338] and Theorem 3.10 with the convex function $\Phi\left(e^{\phi}\right)$ where $\phi$ is the function $t \rightarrow \log \left\{c+\left(b+e^{r t}\right)^{\frac{1}{r}}\right\}$, $\mathrm{t} \varepsilon \mathrm{R}$, which is easily seen to be strictly convex and increasing.

The following theorem is an extension of Theorem 3.10 for integrable functions which are not necessarily non-negative or non-positive. It also contains Theorem 4.1 as a particular case, via [10, Theorem 3.3, p. 1338]. 
THEOREM 6.2. Suppose $\mathrm{f}, \mathrm{g} \in \mathrm{L}^{1}(\mathrm{X}, \mu)$. If $\log ^{+}\left[\mathrm{b}+\left(\delta_{f \delta_{g}}\right)^{+}\right] \varepsilon \mathrm{L}^{1}([0, a], \mathrm{m}) \quad$ (respectively $\left.\log ^{+}\left[\mathrm{b}+(\mathrm{fg})^{+}\right] \varepsilon \mathrm{L}^{1}(\mathrm{X}, \mu)\right)$ for some $\mathrm{b} \geqslant 0$, then

$\log \left[b+(f g)^{+}\right] \prec \prec \log \left[b+\left(\delta_{f} \delta_{g}\right)^{+}\right] \quad$ (respectively $\left.\log \left[b+\left(\delta_{f^{l} g}\right)^{+}\right] \prec<\log \left[b+(f g)^{+}\right]\right)$.

If $b>0$ and if $\log \left[b+\left(\delta_{f} \delta_{g}\right)^{+}\right] \varepsilon L^{1}([0, a], m)$ (respectively $\log \left[b+(f g)^{+}\right]$ $\left.\varepsilon L^{1}(X, \mu)\right)$, then

$\log \left[b+(f g)^{+}\right] \prec \log \left[b+\left(\delta_{f} \delta_{g}\right)^{+}\right]$(respectively

$\left.\log \left[b+\left(\delta_{f l}\right)^{+}\right] \prec \log \left[b+(f g)^{+}\right]\right)$if and only if $(f g)^{+} \sim\left(\delta_{f} \delta_{g}\right)^{+}$(respectively $\left.\left(\delta_{f l g}\right)^{+} \sim(f g)^{+}\right)$.

PROOF. For $b>0$, the result can be obtained from Theorem 3.10 and 6.1 as in Theorem 4.1. The case that $b=0$ then follows from the case that $b>0$ by approximations.

COROLLARY 6.3. Suppose $\mathrm{f}, \mathrm{g} \in \mathrm{L}^{1}(\mathrm{X}, \mu)$. If $\log ^{+}\left[b+\left(\delta_{f^{l}}\right)^{-}\right] \varepsilon L^{1}([0, a], m) \quad$ (respectively $\left.\log ^{+}\left[\mathrm{b}+(\mathrm{fg})^{-}\right] \varepsilon \mathrm{L}^{1}(\mathrm{X}, \mu)\right)$ for some $\mathrm{b}>0$, then $\log \left[\mathrm{b}+(\mathrm{fg})^{-}\right] \ll<\log \left[\mathrm{b}+\left(\delta_{\mathrm{f}} \mathrm{g}\right)^{-}\right] \quad$ (respectively $\left.\log \left[b+\left(\delta_{f} \delta_{g}\right)^{-}\right] \prec \prec \log \left[b+(f g)^{-}\right]\right)$.

If $b>0$ and if $\log \left[b+\left(\delta_{f l}\right)^{-}\right] \varepsilon L^{1}([0, a], m)$ (respectively $\left.\log \left[\mathrm{b}+(\mathrm{fg})^{-}\right] \varepsilon \mathrm{L}^{1}(\mathrm{X}, \mu)\right)$, then $\log \left[b+(f g)^{-}\right] \prec \log \left[b+\left(\delta_{f l}\right)^{-}\right] \quad$ (respectively $\log \left[b+\left(\delta_{f} \delta_{g}\right)^{-}\right] \prec \log \left[b+(f g)^{-}\right]$) if and only if $(f g)^{-} \sim\left(\delta_{f} g\right)^{-}$(respectively $\left.\left(\delta_{f} \delta_{g}\right)^{-} \sim(f g)^{-}\right)$.

PROOF. This follows immediately from Theorem 6.2 on replacing $g$ by $-\mathrm{g}$. Using Theorem 6.2 and Corollary 6.3, we can give a further generalization of [6, Theorem 1] for integrable functions which are not necessarily non-positive or nonnegative. This generalization turns out to be of the same form as Theorem 6.1 except 
that $f, g \in L^{1}(X, \mu)$ are now any pair of integrable functions and that $\delta_{f} g, \quad f g$ and $\delta_{f} \delta_{g}$ can now be replaced respectively by either $\left(\delta_{f} g_{g}\right)^{+},(f g)^{+}$and $\left(\delta_{f} \delta_{g}\right)^{+}$ or $\left(\delta_{f} \delta_{g}\right)^{-},(f g)^{-}$and $\left(\delta_{f l}\right)^{-}$.

\section{A CONCLUDING REMARK.}

The particular case that $\mathrm{b}=0$ in Theorem 6.2 deserves to be highlighted as a single theorem to be given below since it not only generalizes Theorem 3.10 but also gives rise to all the results obtained in Sections 4 and 6 via [10, Theorem 3.3, p. 1338] with an appropriate convex function such as $\Phi(t)=\log \left(b+e^{t}\right), t \varepsilon R$, for some $b \geq 0$ in the case of Theorem 6.2.

THEOREM 7.1. If $\mathrm{f}, \mathrm{g} \varepsilon \mathrm{L}^{1}(\mathrm{X}, \mu)$ are such that $\log ^{+}\left[\left(\delta_{\mathrm{f} \delta_{\mathrm{g}}}\right)^{+}\right] \varepsilon \mathrm{L}^{1}([0, \mathrm{a}], \mathrm{m})$ (respectively $\left.\log ^{+}\left[(\mathrm{fg})^{+}\right] \varepsilon \mathrm{L}^{1}(\mathrm{X}, \mu)\right)$,

then

$$
\begin{aligned}
& \log (f g)^{+} \prec<\log \left(\delta_{f} \delta_{g}\right)^{+} \text {(respectively } \\
& \log \left(\delta_{f^{l} g}\right)^{+} \ll<\log (f g)^{+} \text {) }
\end{aligned}
$$

where the spectral inequalities are strong if $f$ and $g$ are either both non-negative or both non-positive.

ACKNOWLEDGEMENTS. The author wishes to express his sincere thanks to the referee for his many helpful suggestions.

The results contained in this paper except for Theorem 6.2, Corollary 6.3 and Theorem 7.1 formed a part of the author's doctoral dissertation, Equimeasurable Rearpangements of Functions with Applications to Analysis, written under the guidance of Professor N.M. Rice and submitted to the Department of Mathematics, Queen's University, Canada in 1972 .

\section{REFERENCES}

1. DAY, P.W. Rearrangement Inequalities, Can. J. Math. XXIV(1972), 930-943.

2. HARDY, G.H., J.E. LITTLEWOOD and G. PÓLYA. Inequalities, Cambridge, 1934 . 
3. LONDON, D. Rearrangement Inequalities Involving Convex Functions, Pac. J. Math. 34(1970), 749-753.

4. LORENTZ, G.G. and T. SHIMOGAKI. Interpolation Theorems for Operators in Function Spaces, J. Func. Analysis 2(1968), 31-51.

5. LUXEMBURG, W.A.J. Rearrangement Invariant Banach Function Spaces, Queen's Papers in Pure and Applied Mathematics, 10(1967), 83-144.

6. HEWITT, E. and K. STROMBERG. Real and Abstract Analysis, Springer-Verlag, New York, 1965.

7. CHONG, K.M. Spectral Orders, Uniform Integrability and Lebesgue's Dominated Convergence Theorem, Trans. Amer. Math. Soc. 191(1974), 395-404.

8. HARDY, G.H., J.E. LITTLEWOOD and G. POLYA. Some Simple Inequalities Satisfied by Convex Functions, Mess. of Math. 프(1929), 145-152.

9. CHONG, K.M. and N.M. RICE. Equimeasurable Rearrangements of Functions, Queen's Papers in Pure and Applied Mathematics, No. 28, 1971.

10. CHONG, K.M. Some Extensions of a Theorem of Hardy, Littlewood and Pólya and Their Applications, Can. J. Math. XXVI (1974), 1321-1340. 


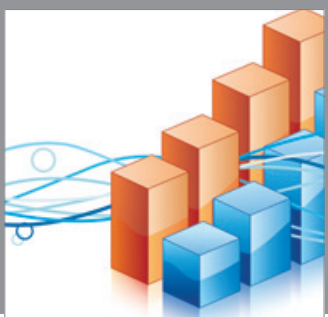

Advances in

Operations Research

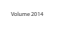

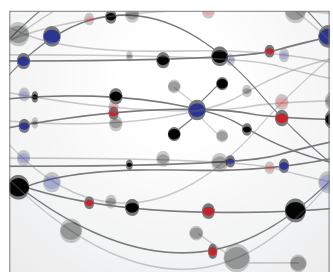

\section{The Scientific} World Journal
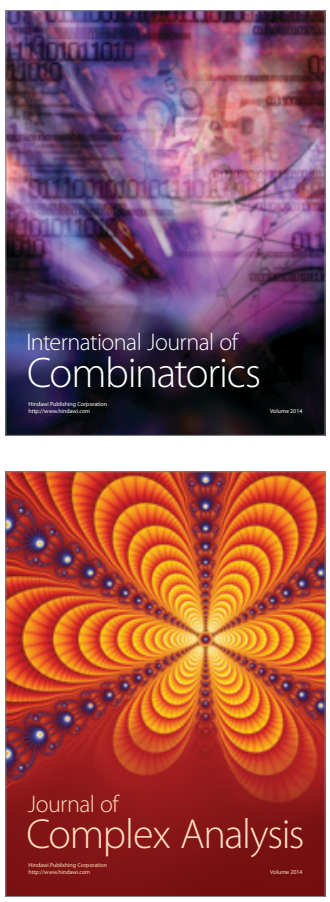

International Journal of

Mathematics and

Mathematical

Sciences
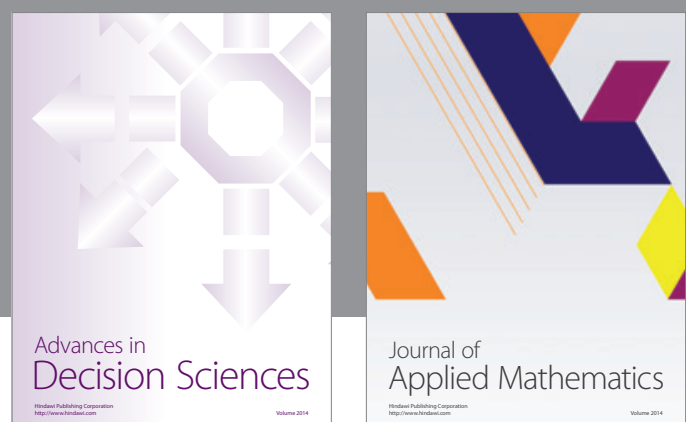

Journal of

Applied Mathematics
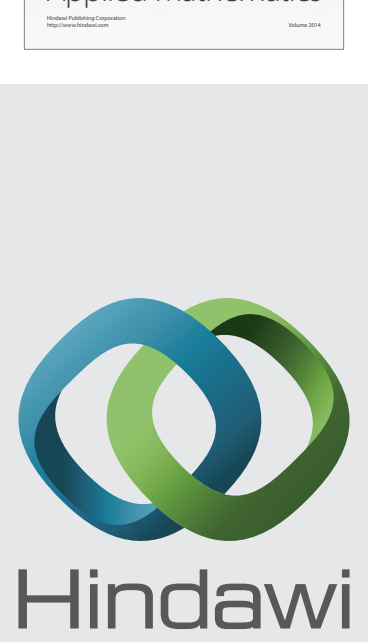

Submit your manuscripts at http://www.hindawi.com
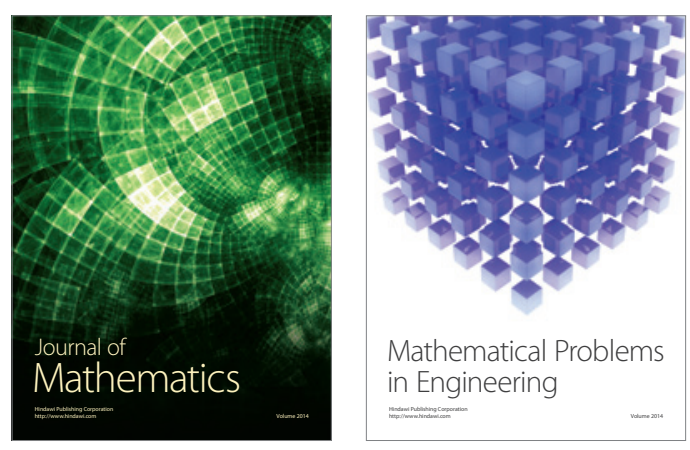

Mathematical Problems in Engineering
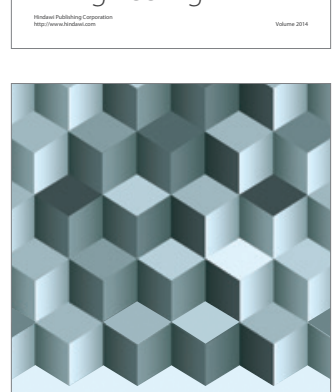

Journal of

Function Spaces
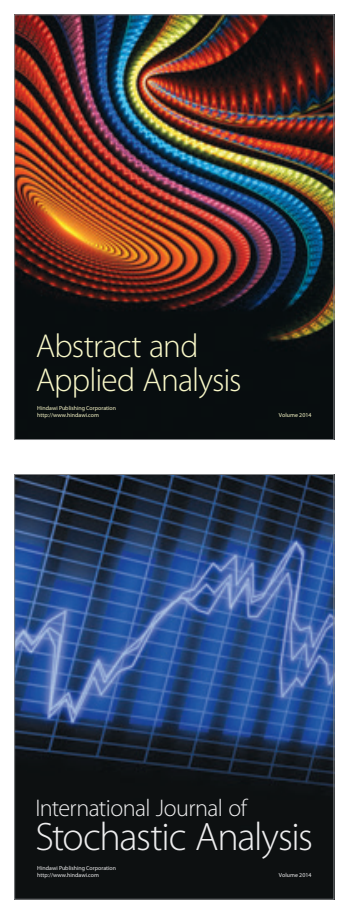

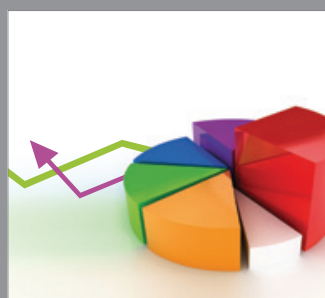

ournal of

Probability and Statistics

Promensencen
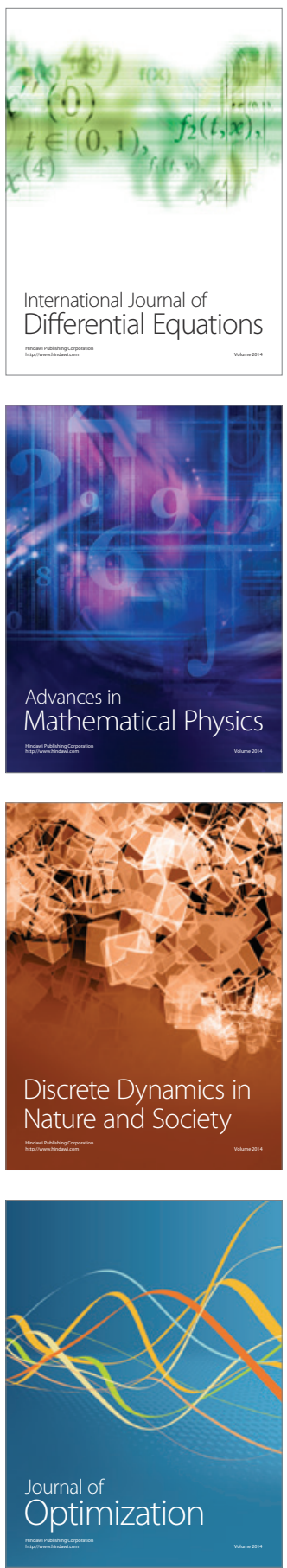